\title{
Влияние примеси самария на структуру и морфологию поверхности халькогенидного стеклообразного полупроводника $\mathrm{Se}_{95} \mathrm{Te}_{5}$
}

\author{
(C) С.И. Мехтиева, С.У. Атаева , А.И. Исаев, В.З. Зейналов \\ Институт фризики им. Г.М. Абдуллаева Национальной академии наук Азербайджана, \\ Az-1143 Баку, Азербайджан \\ E-mail: seva_atayeva@mail.ru
}

(Получена 3 марта 2016 г. Принята к печати 11 октября 2016 г.)

\begin{abstract}
Методами рентгеновской дифракции и атомно-силовой микроскопии исследованы структура и морфология поверхности пленок $\mathrm{Se}_{95} \mathrm{Te}_{5}$, а также влияние на них легирования самарием. С использованием параметров первого резкого дифракционного максимума (FSDP), наблюдаемого в картинах распределения интенсивности дифракции рентгеновских лучей, определены численные значения параметров локальной структуры, в частности „квазиперио““ флуктуаций плотности, длина корреляции, диаметры нанопустот. Кроме того, определены численные значения амплитудных параметров шероховатости поверхности. Установлено, что с увеличением процентного содержания примеси самария наблюдается рост разупорядочения в атомной структуре и рост неоднородностей на поверхности исследуемых пленок.
\end{abstract}

DOI: $10.21883 /$ FTP.2017.06.44561.8228

\section{1. Введение}

Халькогенидные стеклообразные полупроводники (ХСП) с большим успехом применяются в различных приборах микро-, нано- и оптоэлектроники - в оптических запоминающих устройствах, планарных волноводах, волоконных усилителях, оптических переключателях, лазерах и т. д. [1-6], и со временем расширяются области применения указанных материалов. Этому способствует уникальность их электронных свойств, в частности прозрачность в видимой и ближней инфракрасной (ИК) областях, высокая фоточувствительность, оптическая нелинейность, такие параметры, как показатель преломления света, край оптического поглощения.

Известно, что в аморфных полупроводниках с небольшими отклонениями сохраняется ближний порядок (short-range order, SRO) в расположении атомов (координационное число, тип атомов, окружающих данный атом, длины связей и валентные углы), свойственный соответствующим кристаллическим аналогам. Эти отклонения приводят к нарушению строгой периодичности в расположении атомов аморфных полупроводников, т.е. к отсутствию дальнего порядка (long-range order, LRO). Однако в аморфных полупроводниках, в том числе и в ХСП, существуют определенные закономерности во взаимном расположении атомов, межатомные корреляции на расстояниях, превышающих радиус второй координационной сферы, т. е. за пределами области ближнего порядка, - средний порядок (medium-range order, $\mathrm{MRO})$. Эти области играют решающую роль в управлении электронными свойствами, т.е. почти заменяют элементарную кристаллическую решетку, присущую кристаллам. Изменяя параметры указанных областей, можно повлиять на физические свойства, в том числе на электронные. Исследования показали, что параметры областей MRO удается изменить в результате изменения химического состава и легирования атомами редкоземельных элементов [7-10].
Выбор состава $\mathrm{Se}_{95} \mathrm{Te}_{5}$ в качестве объекта исследования обусловлен тем обстоятельством, что замена части атомов селена атомами теллура приводит к модификации цепочечно-кольцевой структуры селена, т.е. способствует частичному разрушению колец $\mathrm{Se}_{8}$ и сокращению длины цепочечных молекул. Использование самария для легирования связано с тем, что самарий как химически активный элемент, проявляя валентность 2, 3 и участвуя как положительный ион, может образовывать новые структурные элементы с атомами селена, теллура и способствовать тем самым модификации структуры. Таким образом, использование в качестве добавки теллура и легирующего элемента самария должно приводить к изменению структуры и концентрации заряженных дефектов, что позволит воздействовать на его электронные свойства. Последнее поможет найти пути успешного практического применения указанного материала и расширения области его использования.

Настоящая работа посвящена исследованию структуры и морфологии поверхности аморфных пленок $\mathrm{Se}_{95} \mathrm{Te}_{5}$ и влияния на них легирования самарием с применением методов рентгеновской дифракции и атомно-силовой микроскопии (АСМ). Указанные исследования позволят проследить за изменением локальной структуры, как в объеме, так и на поверхности исследуемых материалов, т.е. установить взаимосвязь между изменениями в структуре и рельефе поверхности, что важно для выяснения механизма влияния примесей и установления способов получения высококачественных пленок.

\section{2. Методика эксперимента и изготовление образцов}

Синтез чистого $\mathrm{Se}_{95} \mathrm{Te}_{5}$, а также $\mathrm{Se}_{95} \mathrm{Te}_{5}$ с примесью самария (0.25-1 ат\%) осуществлен в следующей последовательности. Особо чистые элементарные вещества в соответствующих атомных концентрациях помещались 
в кварцевые ампулы, после откачивания воздуха до давления $10^{-3}$ мм рт. ст. в течение 3 ч нагревались до температуры $900-950^{\circ} \mathrm{C}$ и выдерживались около 6 ч при этой температуре. С целью обеспечения однородности образцов синтезы проведены во вращающейся печи, а охлаждение - в режиме выключенной печи. Примесь самария вводилась в процессе синтеза. Пленки толщиной 3 мкм, используемые в исследованиях, получены термическим испарением на стеклянную подложку в вакууме (при давлении $10^{-3}$ мм рт. ст.). Исследования агрегатного состояния и структуры осажденных пленок проведены рентгеноструктурным анализом на порошковом дифрактометре D8 ADVANCE фирмы Брукер (Германия) в режиме 40 кВ, $40 \mathrm{MA}$, в диапазоне углов $5<2 \theta<80^{\circ}$, длина волны излучения $\lambda=1.54185 \AA$. Дифракционные картины анализировались аппроксимацией их лоренцевыми функциями с использованием метода двойного дифференцирования максимума, и применялась специальная программа для определения таких параметров дифракционного максимума, как угловое положение $(2 \theta)$ и ширина, соответствующая половине максимума (full width at half maximum, FWHM).

Исследования морфологии поверхности полученных пленок проведены с помощью атомно-силового микроскопа марки AIST-NT (Tokyo Instr., Japan). ACM-исследования образцов проводились в полуконтактном резонансном режиме. Поверхности образцов с размерами $2 \times 2$ мм очищены с помощью чистого азота и помещены на держатель образца АСМ. Размер сканированной области составлял $\sim 1$ мкм $^{2}$.

\section{3. Экспериментальные результаты и их обсуждение}

На рис. 1 показаны кривые дифракции рентгеновских лучей в пленках исследованных материалов, причем широкие максимумы указывают на их аморфность. Как видно, на картине распределения интенсивности дифракции рентгеновских лучей в исследуемых ХСП, как и в большинстве других стекол [11-18], наблюдается узкий максимум, так называемый первый резкий дифракционный максимум (first sharp diffraction peak, FSDP), который отличается от других аномальной зависимостью от температуры и давления [13,14]. FSDP на кривых интенсивности дифракции рентгеновских лучей в ХСП, как правило, связывают с существованием упорядочения в масштабах среднего порядка [12,16-18]. Для стеклообразных материалов с тетраэдрическими структурными единицами Эллиоттом была предложена пустотнокластерная модель [19,20]. Согласно этой модели, в таких материалах группы атомов, образующие кластеры, отделяются друг от друга пустотами или областями с пониженной атомной плотностью. Согласно [21], для большинства ХСП приемлема пустотно-кластерная модель. В связи с взаимным отталкиванием электронов уединенных пар на атомах халькогенов они имеют низкую плотность упаковки, и наличие пустот является

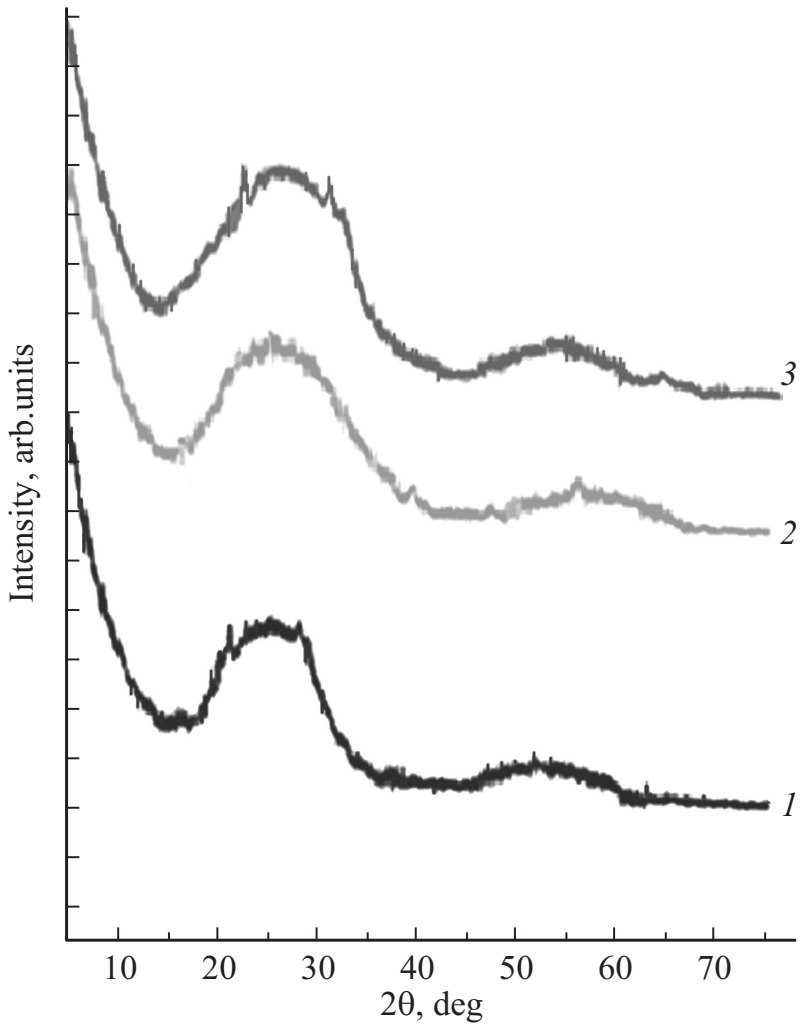

Рис. 1. Рентгенодифракционные картины пленок составов $\mathrm{Se}_{95} \mathrm{Te}_{5}$ (кривая 1), $\mathrm{Se}_{95} \mathrm{Te}_{5}+0.25 \mathrm{aT} \% \mathrm{Sm}$ (кривая 2) и $\mathrm{Se}_{95} \mathrm{Te}_{5}+1$ aт\% $\mathrm{Sm}$ (кривая 3 ).

характерной особенностью их структуры. В связи с этим анализ результатов по дифракции рентгеновских лучей в области, соответствующей FSDP, проводился в рамках наноструктурных особенностей халькогенидных стекол. Параметры структуры, т. е. $R-$ „квазипериод“ структуры или флуктуации плотности атомов [19-21], повторяемость которой в некоторой области корреляции может обусловливать появление FSDP, $L$ - длина корреляции (размеры областей MRO), т. е. размер области, в которой поддерживается периодичность флуктуации атомов, были вычислены согласно формулам [20]

$$
\begin{aligned}
& R \approx 2 \pi / Q_{1}, \\
& L \approx 2 \pi / \Delta Q_{1},
\end{aligned}
$$

где $Q_{1}-$ величина вектора рассеяния, соответствующая положению FSDP и определяемая формулой $Q_{1}=4 \pi \sin \theta / \lambda, \Delta Q_{1}$ - ширина, соответствующая половине амплитуды максимума FSDP. Полученные значения параметров, характеризующих FSDP и структуру исследованных пленок, приведены в табл. 1. Используя формулу, предложенную в $[17,18]$ и связывающую положение FSDP $Q_{1}$ с диаметром нанопустот $(D)$,

$$
Q_{1}=2.3 \pi / D \text {, }
$$

мы оценили значения $D$. Полученные результаты также представлены в табл. 1. 
Таблица 1. Значения параметров ближнего и среднего порядков чистого и легированного самарием ХCП состава $\mathrm{Se}_{95} \mathrm{Te}_{5}$

\begin{tabular}{l|l|c|c|c|c|c|c}
\hline \multicolumn{1}{c|}{ ХСП } & $2 \theta,{ }^{\circ}$ & FWHM, $^{\circ}$ & $Q_{1}, \AA^{-1}$ & $\Delta Q_{1}, \AA^{-1}$ & $R, \AA$ & $L, \AA$ & $D, \AA$ \\
\hline $\mathrm{Se}_{95} \mathrm{Te}_{5}$ & 22.129 & 7.863 & 1.56 & 0.558 & 4.02 & 12.94 & 4.63 \\
$\mathrm{Se}_{95} \mathrm{Te}_{5}+0.25 \mathrm{aT} \% \mathrm{Sm}$ & 22.109 & 10.048 & 1.562 & 0.713 & 4.07 & 8.8 & 4.62 \\
$\mathrm{Se}_{55} \mathrm{Te}_{5}+1 \mathrm{aT} \% \mathrm{Sm}$ & 26.65 & 10.83 & 1.877 & 0.769 & 3.35 & 8.16 & 3.85
\end{tabular}

Таблица 2. Значения параметров шероховатости поверхности пленок чистого и легированного самарием ХСП состава $\mathrm{Se}_{95} \mathrm{Te}_{5}$

\begin{tabular}{l|l|c|c}
\hline \multicolumn{1}{c|}{ ХСП } & \multicolumn{1}{|c|}{$\mathrm{Se}_{95} \mathrm{Te}_{5}$} & $\mathrm{Se}_{95} \mathrm{Te}_{5}+0.25 \mathrm{aT}_{0} \mathrm{Sm}$ & $\mathrm{Se}_{95} \mathrm{Te}_{5}+1 \mathrm{aT} \% \mathrm{Sm}$ \\
\hline$R_{a}, \mathrm{HM}$ & 0.270729 & 0.381862 & 1.12372 \\
$R_{q}, \mathrm{HM}$ & 0.367469 & 0.495805 & 1.65846 \\
$R_{s k}, \mathrm{HM}$ & 1.3849 & 0.947744 & 2.02772 \\
$R z_{\text {max }}, \mathrm{HM}$ & 3.74495 & 6.34558 & 22.6086 \\
$R z_{\text {av }}, \mathrm{HM}$ & 0.962931 & 1.38118 & 4.82635
\end{tabular}

Авторами [16] проведен анализ формул, предложенных различными исследователями [22,23] для определения расстояний межатомных корреляций или расстояний между пустотами, и показано, что, во всех случаях полученные результаты близки к значениям диаметра пустот, полученным из формулы (3).

На рис. 2 представлены двухмерные и трехмерные ACМ-изображения пленок $\mathrm{Se}_{95} \mathrm{Te}_{5}$, легированных самарием. Как видно из рисунка, особенности АСМ-изображения претерпевают существенные изменения в результате легирования. Параметры, характеризующие морфологические особенности поверхности, представлены в табл. 2.

В таблице $R_{a}$ - среднеарифметическая шероховатость, т.е. среднее арифметическое абсолютных значений вертикальных отклонений от средней линии, $R_{q}-$ среднеквадратичная шероховатость, являющаяся квадратным корнем из среднего арифметического квадратов вертикальных отклонений от опорной линии. Сред-
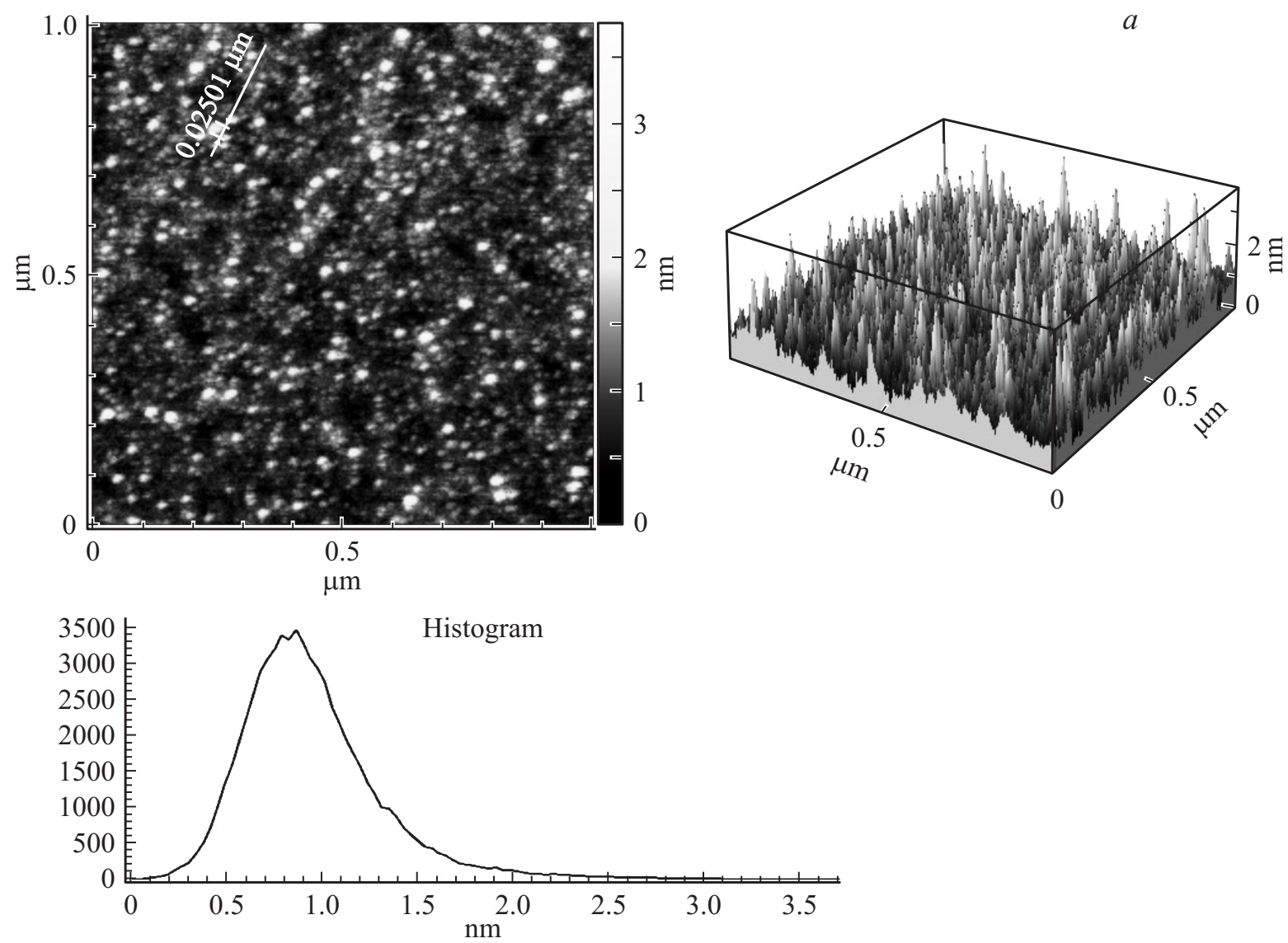

Рис. 2. Двухмерные и трехмерные АСМ-изображения рельефа поверхности и гистограмма распределения максимумов пленок $\mathrm{Se}_{95} \mathrm{Te}_{5}$, не легированных и легированных самарием: $a-\mathrm{Se}_{95} \mathrm{Te}_{5}, b-\mathrm{Se}_{95} \mathrm{Te}_{5}+0.25 \mathrm{aT} \% \mathrm{Sm}, c-\mathrm{Se}_{95} \mathrm{Te}_{5}+1 \mathrm{aT} \% \mathrm{Sm}_{\text {. }}$ 

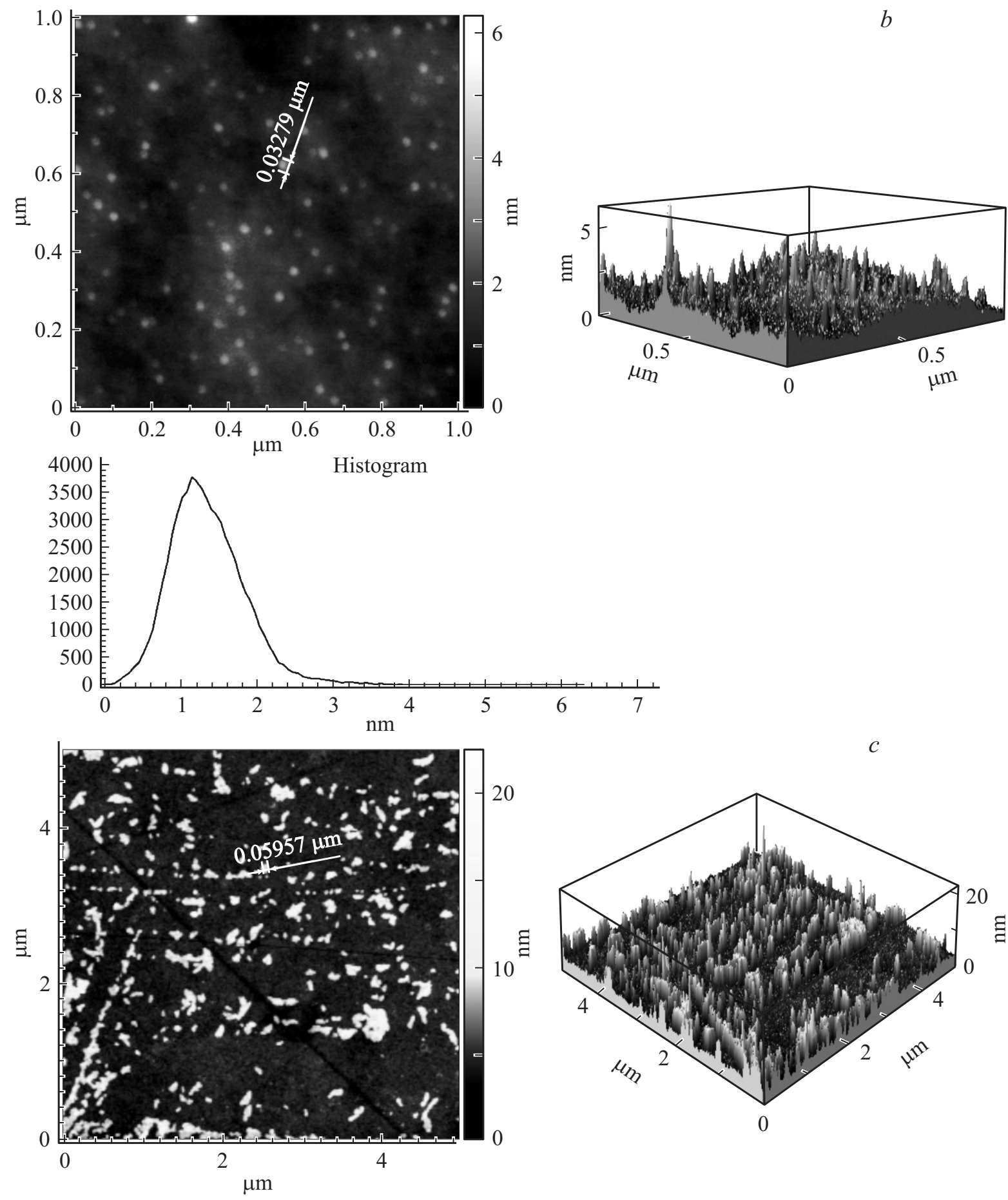

$c$

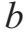

$b$

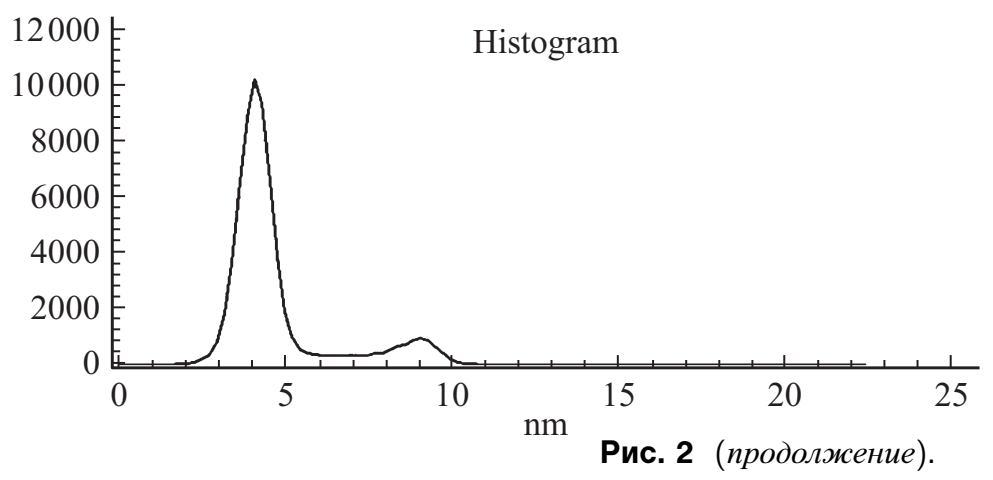


неквадратичная шероховатость характеризует ширину гистограммы распределения высот - чем больше $R_{q}$, тем шире гистограмма и тем грубее поверхность, что и наблюдается в наших исследованиях с увеличением уровня легирования. $R_{s k}-$ коэффициент асимметрии поверхности, описывает асимметрию гистограммы распределения высот; $R_{k u}$ - эксцесс поверхности, описывающий „островершинность“ топографии поверхности; $R_{s k}=0$ и $R_{k u}=3$ свидетельствуют о симметричности распределения высот, при этом имеются равные количества локальных максимумов и минимумов с определенной высотой выше и ниже средней линии. Для высоких отрицательных значений $R_{s k}$ и низких значений эксцесса $\left(R_{k u}<3\right)$ характерно гауссово распределение, для положительных $R_{s k}$ и высоких значений эксцесса $\left(R_{k u}>3\right)$ характерно большее число локальных минимумов ниже средней линии по сравнению с гауссовым распределением. В исследованных нами пленках выполняется последнее условие, что свидетельствует о большом числе локальных минимумов ниже средней линии. $R z_{\max }-$ максимум шероховатости, показывающий максимальный перепад высот между самой верхней и нижней точками поверхности профиля; $R z_{\text {av }}$ - наиболее часто встречающаяся высота.

Как видно из рисунков и таблиц, и структура, и морфология поверхности пленок $\mathrm{Se}_{95} \mathrm{Te}_{5}$ заметно изменяются при легировании. Такие изменения, происходящие в структуре и морфологии поверхности, можно объяснить в рамках модели Эллиотта и модели собственных заряженных дефектов $\left(D^{+}\right.$и $\left.D^{-}\right)$с учетом особенностей распределения атомов самария и их химической активности. Предполагается, что атомы самария в малых концентрациях в основном заполняют существующие пустоты и как атомы химически активного элемента образуют химические связи со всеми элементами, входящими в аморфную матрицу. Часть атомов самария в аморфной матрице проявляется в виде положительных ионов и накапливается вокруг $D^{-}$-центров. Таким образом, в результате легирования, особенно при больших концентрациях, увеличивается степень неупорядоченности. Такое изменение структуры можно заметить как на рисунках, касающихся дифракции рентгеновских лучей, АСМ-изображениях поверхности, так и в значениях параметров, характеризующих наноструктуру и морфологию поверхности. Вследствие этого частично нарушается повторяемость флуктуации плотности, уменьшается амплитуда FSDP, а также длина корреляции (размеры областей MRO). Из табл. 2 и рис. 2 (из трехмерных АСМ-изображений) видно, что с легированием значения параметров, характеризующих амплитуды шероховатости $\left(R_{a}, R_{q}, R z_{\max }, R z_{\text {av }}\right)$, увеличиваются. Кроме этого, как видно из рис. 2 , где представлены двумерные АСМ-изображения поверхности, с ростом концентрации атомов самария увеличиваются размеры морфологических неоднородностей, что связано с накоплением ионов самария вокруг $D^{-}$-центров и образованием новых структурных элементов с участием атомов самария.

\section{4. Заключение}

Методами рентгеновской дифракции и атомносиловой микроскопии исследована структура и морфология поверхности пленок $\mathrm{Se}_{95} \mathrm{Te}_{5}$, а также влияние на них легирования самарием. Для всех образцов на картине распределения интенсивности дифракции рентгеновских лучей наблюдается FSDP, что связывается с существованием упорядочения в масштабах среднего порядка. Интерпретация полученных результатов проведена в рамках пустотно-кластерной модели Эллиотта и модели заряженных дефектов, определены параметры локальной структуры, в частности „квазипериод“ флуктуации плотности, длина корреляции (размеры областей $\mathrm{MRO})$, диаметры нанопустот. Кроме этого определены амплитудные параметры шероховатости $\left(R_{a}, R_{q}, R z_{\max }\right.$, $\left.R z_{\text {av }}\right)$, коэффициенты асимметрии $\left(R_{s k}\right)$ и эксцесс $\left(R_{\mathrm{ku}}\right)$ поверхности. Изменения, происходящие в картинах распределения интенсивности рентгеновских лучей и ACM-изображениях, а также в численных значениях параметров при легировании, объяснены особенностями распределения атомов самария в аморфной матрице, проявлением их в виде положительных ионов и их химической активностью.

Авторы статьи выражают благодарность сотрудникам Инновационного сектора И.Р. Амирасланову и Е.Н. Алиевой за помощь, оказанную при проведении экспериментов.

\section{Список литературы}

[1] I. Aggarwal, J. Sanghera. Optoelectron. Adv. Mater., 4 (3), 665 (2002)

[2] J. Viens, C. Meneghini, A. Villeneuve, T. Galstian, E. Knystautas, M. Duguay, K. Ricardson, T. Cardinal. J. Lightwave. Technol., 17, 1184 (1999).

[3] Y. Ruan, W. Li., R. Jarvis et al. Opt. Express, 12, 5140 (2004).

[4] A. Kovalskiy, M. Vlcek, H. Jain, A. Fiserova, C.M. Waits, M. Dubey. J. Non-Cryst. Sol., 352, 589 (2006).

[5] A. Ozols, D. Saharovs, M. Reinfelde. J. Non-Cryst. Sol., 352, 2652 (2006).

[6] X. Zhang, H. Ma, J. Lucas. J. Optoelectron. Adv. Mater., 5, 1327 (2003).

[7] С.И. Мехтиева, А.И. Исаев, С.У. Атаева, В.З. Зейналов. ФТП, 48 (9), 1182 (2014).

[8] Р.И. Алекберов, А.И. Исаев, С.И. Мехтиева, Г.А. Исаева. ФТП, 48 (6), 818 (2014).

[9] Р.И. Алекберов, С.И. Мехтиева, Г.А. Исаева, А.И. Исаев. ФТП, 48 (6), 823 (2014).

[10] R.I. Alekberov, A.I. Isayev, S.I. Mekhtiyeva. Chalcogenide Lett., 10 (9), 335 (2013).

[11] Электронные явления в халькогенидных стеклообразных полупроводниках, под ред. К.Д. Цэндина (СПб., Наука, 1996).

[12] F. Sava, A. Lorinczi, M. Popescu, G. Sokol, E. Axente, I.N. Mihailscu, M. Nistor. J. Optoelectron. Adv. Mater., 8 (4), 1367 (2006).

[13] L.E. Busse, S.R. Nagel. Phys. Rev. Lett., 41, 1848 (1981).

[14] L.E. Busse. Phys. Rev. Lett. B, 29, 3639 (1984). 
[15] K. Tanaka. Philos. Mag. Lett., 57, 183 (1988).

[16] T.S. Kavetskyy, O.I. Shpotyuk, V.T. Boyko. J. Phys. Chem. Solids, 68, 712 (2007).

[17] T.S. Kavetskyy, O.I. Shpotyuk. J. Optoelectron. Adv. Mater., 7, 2267 (2007).

[18] O.I. Shpotyuk, A. Kozdras, T.S. Kavetskyy, J. Filipecki. J. NonCryst. Sol., 352, 700 (2006).

[19] S.R. Elliott. Phys. Rev. Lett., 67, 711 (1991).

[20] S.R. Elliott. J. Non-Cryst. Sol., 182, 40 (1995).

[21] Э.А. Сморгонская, К.Д. Цэндин. В кн.: Электронные явления в халькогенидных стеклообразных полупроводниках, под ред. К.Д. Цэндина (СПб., Наука, 1996) с. 9.

[22] P.H. Gaskell. J. Non-Cryst. Sol., 351, 1003 (2005).

[23] O.P. Rachek. J. Non-Cryst. Sol., 352, 3781 (2006).

Редактор Л.В. Шаронова

\section{Influence of samarium impurity on structure and surface morphology of $\mathrm{Se}_{95} \mathrm{Te}_{5}$ chalcogenide glassy semiconductor}

S.I. Mekhtiyeva, S.U. Atayeva, A.I. Isayev, V.Z. Zeynalov

Abdullayev Institute of Physics,

Azerbaijan National Academy of Sciences,

Az-1143 Baku, Azerbaijan

Abstract The structure and the surface morphology of $\mathrm{Se}_{95} \mathrm{Te}_{5}$ chalcogenide glassy semiconductor films and influence of samarium impurity on them have been investigated by $X$-ray diffraction and atomic-force microscopy methods. By using the data from the first sharp diffraction maximum (FSDP), observed in the pattern of distribution of $X$-ray diffraction intensity the numerical values of the local structure parameters, such as density fluctuations „quasi-period“", the correlation length (MRO field size), the nanocavities diameter were defined. In addition, the numerical values of amplitude roughness parameters, were determined. It was established that increase in the percentage of samarium impurity the disorder growth in the atomic structure and irregularities on the investigated films surface observed. 